Patient Educ Couns. 2013 June ; 91(3): 372-377. doi:10.1016/j.pec.2013.01.011.

\title{
A qualitative study of women's perceptions of provider advice about diet and physical activity during pregnancy
}

\author{
Renée M. Ferrari, \\ Cecil G. Sheps Center for Health Services Research, 725 Martin Luther King, Jr. Blvd., Chapel \\ Hill, NC 27599-7590, USA

\begin{abstract}
Anna Maria Siega-Riz, Departments of Nutrition and Epidemiology, The University of North Carolina at Chapel Hill, Chapel Hill NC, USA
\end{abstract}

Kelly R. Evenson, Department of Epidemiology, The University of North Carolina at Chapel Hill, Chapel Hill NC, USA

\author{
Merry-K. Moos, and \\ Department of Obstetrics and Gynecology, School of Medicine, The University of North Carolina \\ at Chapel Hill, Chapel Hill NC, USA
}

\section{Kathryn S. Carrier \\ Department of Epidemiology, The University of North Carolina at Chapel Hill, Chapel Hill NC, USA}

\begin{abstract}
OBJECTIVE-The purpose of this qualitative study was to gather insights into pregnant women's experiences with provider advice about diet and physical activity.

METHODS-We conducted a series of 13 focus groups with a total of 58 pregnant African American, Caucasian, and Hispanic women of varying body sizes. Statements were independently coded, reduced, and then reconstructed to identify overarching themes with the assistance of ATLAS/ti software.

RESULTS-Mean gestational age at the time of the focus groups was 30 weeks. Women commonly reported overwhelming and confusing diet advice and a paucity of physical activity advice that was largely limited to walking. Many reported following advice; when advice was not followed, it was because women disagreed with it or simply did not want to do it.
\end{abstract}

CONCLUSION-Women would benefit from more clear guidance from physicians and other providers regarding dietary choices and physical activity in pregnancy.

(C) 2013 Elsevier Ireland Ltd. All rights reserved.

Corresponding author at: Renée Ferrari, The University of North Carolina at Chapel Hill, Cecil G. Sheps Center for Health Services Research, 725 Martin Luther King, Jr. Blvd., Chapel Hill, NC 27599-7590, USA, rferrari@ unc.edu; Phone: 919-843-1341; Fax: 919-966-5764.

Publisher's Disclaimer: This is a PDF file of an unedited manuscript that has been accepted for publication. As a service to our customers we are providing this early version of the manuscript. The manuscript will undergo copyediting, typesetting, and review of the resulting proof before it is published in its final citable form. Please note that during the production process errors may be discovered which could affect the content, and all legal disclaimers that apply to the journal pertain. 
PRACTICE IMPLICATIONS-Providers should make dietary and physical activity advice in pregnancy more clear and individualized and offer such guidance multiple times throughout pregnancy.

\section{Introduction}

Poor diet and low physical activity levels are prevalent across the United States (US) and other developed countries [1-4] and are of concern for pregnant women as well. [5-7] In the US, established recommendations for diet and physical activity during pregnancy advise women to consume an additional 340-450 kilocalories (kcal) daily in accordance with US healthy eating guidelines, take appropriate nutritional supplements, limit caffeine, and avoid alcohol, tobacco, and illicit drugs. [8,9] The guidelines highlight the importance of individualized recommendations. [8] Physical activity guidelines state that, "in the absence of either medical or obstetric complications, 30 minutes or more a day on most, if not all, days of the week is recommended for pregnant women." [10]

Poor nutrition during pregnancy has been linked to birth defects, [11] preterm delivery, [12] small-for-gestational age infants, [13] and postpartum weight retention. [14,15] Recreational physical activity in pregnancy is believed to impart the same benefits as exercise before pregnancy $[16,17]$ and may have the added benefit of preventing or ameliorating gestational diabetes [18-19] and reducing the risk of preeclampsia [19-21] and preterm birth. [22] Exercise improves energy levels, decreases physical discomfort, and eases labor and postpartum recovery. [23]

Most pregnant women do not follow dietary guidelines, [24] consuming too much fat and sugar (in particular, soft drinks) and not enough vegetables, fruits, grains, and protein. [6,24-25] In addition, few meet physical activity recommendations [7,26-27] and activity decreases as pregnancy progresses. [7,22,27-28] Pregnant women who receive targeted counseling demonstrate improved nutrition and activity levels compared to those without such advice. [29] However, women's poor dietary intake and low activity levels suggest that they are not receiving, not hearing, or not following advice.

The purpose of this study was to examine pregnant women's experiences with provider advice to inform future interventions aimed at improving diet and physical activity in pregnancy. Toward that end, we used an applied research design [30] and gathered data via a series of focus groups in which pregnant women were queried about their advice experiences.

\section{Methods}

\subsection{Source and population}

The focus groups were part of a larger study examining factors associated with women's diet, physical activity, and weight gain/weight loss behaviors in pregnancy and postpartum. Eligibility included pregnant women who self-identified as Hispanic, Nonhispanic African American, or Nonhispanic White, spoke English or Spanish, were between 18-35 years old and 27-30 weeks of gestation. Women were recruited through newspaper advertisements, posted flyers, and prenatal clinics.

We stratified the focus groups by race/ethnicity [African American, Caucasian, and Hispanic (Spanish speaking)] and self-reported pre-pregnancy body mass index (BMI) ['overweight' $\left(\mathrm{BMI} \geq 26 \mathrm{~kg} / \mathrm{m}^{2}\right.$ ), and 'not overweight' $\left(\mathrm{BMI}<26 \mathrm{~kg} / \mathrm{m}^{2}\right)$ ]. Homogeneity in groups can promote openness. [31] Thus, we stratified by body size recognizing that some women may feel more comfortable talking about weight-related issues among similarly-sized women. 
We further stratified by race/ethnicity, hypothesizing that race, as a marker for common social experiences, might influence women's experiences with advice in pregnancy.

We held two focus groups per race/ethnicity and BMI grouping, with an additional group for African American overweight women to increase the absolute number of women in that stratum. A total of 13 groups were held between March 2003 and September 2004. Informed consent was obtained. The Institutional Review Board of the University of North Carolina at Chapel Hill approved all study protocols.

\subsection{Research design and focus group process}

After consent, participants provided basic demographic information. Hispanic women completed a short acculturation scale that included questions such as language spoken at home and preferred language for viewing media. [32] Participants were offered free childcare and refreshments, reimbursement for travel and parking, and a $\$ 35$ cash incentive upon completion. Groups were 75 minutes in length and facilitated by moderators matched to the focus group population by race/ethnicity. Moderators used a field-tested, standardized open-ended interview guide covering topics such as: habits and attitudes about diet and physical activity; barriers and enablers to healthy eating and physical activity; and strategies for healthy eating and physical activity during pregnancy and postpartum. This guide is available on the Pregnancy, Infection, and Nutrition Study website, http://www.cpc.unc.edu/ projects/pin/design_postpart/docs_postpart/Focus-Group-Guide-may03.pdf.

Focus groups were audio-taped and transcribed verbatim. The Hispanic groups were conducted in Spanish and audio-translated into English in real time by a native Spanish speaker. Afterwards, the translator transcribed the English audiotape and checked that transcript for accuracy against the Spanish audiotape.

\subsection{Analysis}

Data were organized using the qualitative software program ATLAS/ti. Two researchers independently coded each transcript and negotiated any coding discrepancies through discussion. Analysis involved a reduction and reconstruction process to identify overarching themes, whereby researchers read participants' responses to capture their essential meaning; collapsed each response into a code or codes that reflected the comment's essence; displayed that information in a matrix (reduction); reviewed the data for patterns; and ultimately reconstructed the data into broad themes (reconstruction). [33] Researchers independently reviewed the resulting themes for verification [33] across all groups, which included reevaluating quotes against the themes to ensure themes reflected participants' experiences. At the same time researchers noted important differences by race/ethnicity or pre-pregnancy BMI.

\section{Results}

\subsection{Participant characteristics}

One-hundred and seventy-seven women were deemed eligible to participate. Of those, 58 participated in the groups. The most common reasons for non-participation were timing out of the gestational-age window before the focus group was held and being invited but ultimately not attending a group. Table 1 displays focus group details. In three cases, women with a 'not overweight' pre-pregnancy BMI close to 26.0 were included in 'overweight' BMI groups as they were far along in their pregnancies and were unable to wait for a later 'not overweight' focus group. Mean gestational age was 30 weeks with little difference between groups. Compared to their African American and Hispanic counterparts, White women were older and had higher education levels and were married. Less than ten percent 
were unemployed. In contrast, about half of African American and Hispanic participants were married and one-fifth to one-third was unemployed (Table 2).

\subsection{Themes}

\subsubsection{Eating Advice Themes}

Eating advice is overwhelming or not individualized: African American and Caucasian women voiced concerns that diet advice was overwhelming or, particularly in the case of overweight African American participants, not tailored to individual needs. Women commented on the extent of the diet advice: "the list [of what to eat and what not to eat] to me is so huge," (Not overweight, Caucasian) and frequently were unable to recall what the dietary guidelines entailed: "I've heard so many things [about what to eat or not eat], now I can't think of them." (Not overweight, Caucasian)

Several women felt overwhelmed by advice. "You know like you're not allowed to eat sushi and... and these things about fish... keeping up with what you are and aren't supposed to eat is really exhausting." (Not overweight, Caucasian) Another commented: "I find it really hard [following eating advice] and I'm actually not all that good. I like stopped drinking herbal tea entirely because I was like, I have no idea what's in it, hibiscus leaves, are those bad or good, it was too overwhelming...." (Not overweight, Caucasian)

Some women worried that they would be unable to meet all of the dietary requirements: "I feel like there are a lot of dietary demands... I can't drink any juice because it makes me really sick so I always worry about my vitamin $\mathrm{C}$ because it's really hard to get enough without drinking juice." (Not overweight, Caucasian) Another echoed those feelings. "They tell you to eat your greens, eat this - it's like it's too much in that pyramid [the Food Guide Pyramid]... it's too many meats, too many vegetables, too many - I mean, all this stuff, it's too much to eat in one day!" (Overweight, African American)

A concern voiced particularly by overweight African American women was that providers' recommendations were too general and not clearly applicable to them as individuals. "Well, personally, I like to go by stuff that I read more often than the doctors here because they're kind of impersonal...." (Overweight, African American) Referring to nutritionists from the Special Supplementary Food Program for Women, Infants, and Children (WIC), another woman commented on how she did not receive any specific advice and was told to "... follow the pyramid." (Overweight, African American) For one, the generalized recommendations were reason enough not to follow them: “...I don't really pay attention unless it's something you're telling me directly...." (Overweight, African American)

Eating advice is confusing and often changing: Whether overweight or not, African American and Caucasian participants stated repeatedly that dietary information was confusing or frequently changing. One woman asked, "Why do they say don't eat shellfish?" (Not overweight, African American) Another noted that alfalfa sprouts were bad to eat but couldn't remember why, (Not overweight, Caucasian) and another knew she needed to get iron from foods but added, "I don't know what contains iron in food." (Overweight, African American) Others reported confusion: "This is new to me. I don't know what I'm supposed to do. I just know you're not supposed to smoke or drink alcohol, that's all I was told...." (Overweight, Caucasian)

Caucasian women in particular expressed frustration at what they perceived as often changing recommendations: "Every day is a different story about fish and mercury content...." (Not overweight, Caucasian) An overweight woman noted: "I think the list has grown [from her first pregnancy to her second]. I don't remember deli meats and all that 
stuff being on it two years ago." (Overweight, Caucasian) Another participant commented on changing dietary trends: “...I mean carbohydrates, limiting white bread and stuff like that is way out. People are saying that's really, really bad but two years ago that wasn't necessarily true. It was fat. You have to limit fat." (Not overweight, Caucasian)

Hispanic women did not question eating advice: In contrast, Hispanic women expressed little confusion and offered few complaints or comments about the advice they received. When asked what, if any, dietary advice they had received from their health care providers, Hispanic women commonly recited a litany of healthy foods and beverages and a description of what they were told: "They have given me the instruction that I need to eat more fruits, vegetables, not much meat but more vegetables and a lot of fruit...and to drink more water than sodas." (Overweight, Hispanic)

\subsubsection{Physical activity themes}

Women receive little or no advice about physical activity or have to request it: Most women indicated that they received little or no activity advice. When asked if they had received physical activity advice, typical responses included: "No, not really, because they asked what I was doing and once I told them they was like, 'Okay, that's good."' (Overweight, African American), and "Nobody told me nothing [about physical activity]. They gave me some brochures [chuckling] and that's it." (Overweight, African American). One woman was particularly distressed. "No one tells me anything. I am always alone. ...he [the doctor] has only told me that I need to walk. Nothing else." (Overweight, Hispanic)

In the absence of offered advice, some women broached the subject themselves. "I asked, they didn't really say, 'I'm going to tell you all this,' I asked her 'What can I do to exercise?', because I don't want to stop, you know...even when I have the baby I don't want to be looking like Two Ton Tilly, so ... they advised to exercise." (Overweight, African American). Another stated: "My midwives had told me, when I asked the question can I still exercise, they said as long as you were doing it before, then you can do it now...." (Not overweight, Caucasian)

Physical activity advice is generally vague and largely limited to being told to "walk": Women reported receiving advice that was commonly conservative and often vague. The little advice that was received directed women to walk: "From my doctors I got again, walk...." (Overweight, African American); "The midwives did ask ... about exercise... I guess their general recommendation is a lot of walking." (Not overweight, Caucasian). "They say I should walk a lot. Don't lay down all the time." (Not overweight, Hispanic)

Several women reported receiving unclear recommendations that didn't necessarily encourage them to be active, but rather that they could exercise if they wanted, or "as much as they can", if they listened to their own bodies. A typical comment was, "You know [the doctor told me] to just kind of listen to my body and not do anything that I didn't feel comfortable with...." (Not overweight, Caucasian). Others reported more encouraging, though still vague, directives. They stated that they were advised to "...exercise as much as I can within the limits of being pregnant...." (Overweight, African American), and to "...do as much as you could as long as you weren't hurting yourself." (Overweight, African American) and to "...make sure that you're listening to your body ... You can do whatever you feel like and there's no restriction on any type of exercise." (Not overweight, Caucasian)

Other women openly expressed confusion about whose advice to follow or how to interpret it: "I don't know what to follow. I don't know whether to obey what the nurse says about lying down or her [my husband's aunt]." (Not overweight, Hispanic) Another woman noted that, "I would say there's a little bit of confusion over what kind of activity I can do when, 
early in my pregnancy. With my first baby I went skiing, I was like seven weeks pregnant and my doctor was like, "What!" But to me at that point I was like how could I possibly hurt the baby. ...there's some varying advice on what I could do when." (Not overweight, Caucasian)

\subsubsection{Following advice themes}

Many women report following provider advice regarding diet and physical activity during pregnancy: In spite of the confusion or frustration women reported about dietary and physical activity advice, and the lack of physical activity advice, many women stated that they followed advice. Another woman commented that "I follow the recommendations because it makes me feel secure and I'm not going to be worried about what if I eat this or what if I eat that? I just follow the recommendations." (Overweight, Hispanic) Still another stated: "I'm trying to do what's best for my baby [by following dietary advice] ...."

(Overweight, Hispanic) Others commented on physical activity advice, stating that, "It's very important [to follow physical activity advice] because if you get out there and overdo it then, I mean, you can cause problems [for your baby or yourself]." (Not overweight, African American).

Some women followed advice because they valued the provider's opinion. When asked if she followed her provider's advice about diet, one woman responded, "I do I do, because I think they know what they're doing." (Overweight, Caucasian) Another stated that "All the women will tell you the doctor knows more about you and your baby's health too."

(Overweight, Hispanic) Just as often, women gave no reason for following the advice, and said things such as, "The list to me is so huge but like I was told a lot of things that a lot of other pregnant people don't follow but I follow. So if someone tells me no, I won't do it." (Not overweight, Caucasian)

Finally, advice often changed specific behaviors "Sometimes they have recommended some foods. The foods haven't appealed to me but I eat them anyway." (Overweight, Hispanic) Another overweight woman also changed her diet saying she ate "Beans because they have a lot of iron so I should eat a lot of beans. Last month they asked me to eat a lot of iron because the iron was very low in my body." (Overweight, Hispanic) One woman noted that she "... used to drink a lot of sodas. The doctor asked me to stop... Now I drink more water." (Overweight, Hispanic)

When women report not following provider advice about diet or physical activity, it is because they disagreed with the advice, felt they couldn't follow it, or didn't want to Several women reported not following provider advice because they disagreed with it, such as this woman who said, “... whenever I would check my heart rate on my watch or with the clock it would be way higher than what they [health care providers] said the normal range was but I wasn't doing full aerobics. So I guess I didn't take everything to heart that they said." (Not overweight, Caucasian) Another woman stated, "Someone told me...that you've got to eat all the time. I didn't agree with that...." (Not overweight, African American)

The most common reasons women gave for not following advice was that they felt they couldn't follow it or they simply didn't want to. One woman expressed strong feelings against advice she'd received: "I don't want to be told what to eat. I've done it too many times. I don't want to do it anymore." (Overweight, Caucasian) Typical comments included: "Even if they tell you to eat something but you don't feel like it, you just can't eat it." (Overweight, Hispanic); and "I walk of course. But when you feel tired and want to be in bed all the time, what else can you do. So I go to bed." (Not overweight, Hispanic) 


\section{Discussion and Conclusion}

\subsection{Discussion}

The aim of this qualitative study was to elicit from pregnant women their perceptions about provider advice regarding diet and physical activity in pregnancy. The findings verify previous studies and add insight into quantitative research. Importantly, our research adds new knowledge about women's experiences with diet and physical activity, in particular why women choose or decline to follow provider advice.

On the whole, women reported that they received little or no provider advice and when they did, such advice did not meet their needs: dietary advice was either overwhelming or not individually tailored and physical activity advice was rarely given or too conservative. Most stated that they followed provider advice and when they didn't, it was because they disagreed or chose not to.

With regard to dietary advice, African American and Caucasian women, regardless of BMI, echoed similar sentiments. They voiced concerns that advice was not individualized, the amount of eating advice was overwhelming, and that keeping up with changes in recommendations was tiring. In contrast, Hispanic women largely refrained from commenting on advice and were notably less critical. The Hispanic women in our study were all foreign born with low acculturation to US culture. Previous studies of Hispanic culture reported fatalistic beliefs about health - the idea that matters of health are outside their control and are left to higher powers (i.e., God).[34,35] This belief was higher among immigrant Latinos compared to those born in the US. [35] Thus, not challenging provider advice may reflect the cultural belief that provider advice doesn't matter, as it is God's will that will determine outcomes.

Similarly, others have found that pregnant women reported confusing or overwhelming advice. [36] Modern pregnant women receive volumes of advice from various sources during pregnancy, including providers, family members, friends, the Internet, television, and print media. [36,37] Likewise, although the present analysis focused on provider advice, most women reported receiving advice from multiple sources. Women may feel overwhelmed by advice because of the multiple sources of information available to them. [36] Such findings suggest that an important role of the health care provider, then, is to sift through information for women and present them with clear, understandable guidelines.

In our study women commonly reported receiving physical activity advice that was conservative, a finding supported by others. [37] Research on providers indicates that many hold inaccurate beliefs and knowledge about exercise and pregnancy guidelines. [38] Others reported insufficient training, lack of comfort with counseling, and the perception that their advice is ineffective in changing women's behaviors. [39] Thus, providers may not have the knowledge, skills, or confidence needed to effectively counsel pregnant women about physical activity.

Women themselves may not see the value of physical activity. In one study, women rated sleep and rest and relaxation as more important than exercise: $79 \%$ said a good night's sleep was very important, compared to $49 \%$ for rest and relaxation, and only $25 \%$ for exercise. [36] The authors highlight the need to address misconceptions about exercise in pregnancy and to adequately educate about the benefits. This may be challenging if providers don't have the information themselves or are not comfortable encouraging exercise. [39,40] Encouraging greater levels of physically activity in pregnancy may mean increasing provider education, skill, and/or comfort level with advising women to exercise, even vigorously, during pregnancy. [38-41] We speculate that cautious physical activity advice, 
as well as the plethora of eating advice focused on items to avoid, may also reflect a view of pregnancy as a high-risk state, in which any fetal risk, however marginal, is to be avoided regardless of a potential benefit to the mother or her value system. [42] Thus, a paradigm shift in how pregnancy is viewed and how risks are communicated may be necessary precursors to more individualized, evidence-based advice. [42,43].

Some limitations of this study are worth noting. Stratifying by race/ethnicity and prepregnancy body size created six sub-categories which may have interfered with reaching theme saturation; with more groups per category, different themes may have emerged. Similarly, due to invited participants not attending groups, some groups had few participants. Also, participants were not asked to specifically identify the source of advice; it is not known whether the particular type of provider would influence women's experiences with advice. The Hispanic women included in our study had low acculturation and most were Mexican. We do not know if the experiences would be different for US born Hispanic women, those from countries other than Mexico, or those with higher acculturation. We did not collect maternal history characteristics, such as parity, that likely influenced experiences with advice. Thus, we were unable to describe possible associations between such factors and provider advice. Finally, this study was conducted in the United States. It is unclear how these findings might differ if conducted in other countries under different health care systems.

\subsection{Conclusion}

Provider advice, as currently perceived by women, appears insufficient to help pregnant women maintain proper nutrition and physical activity in pregnancy. More research is needed to better understand why women find advice overwhelming at times and at other times report it is not enough, and why physical activity advice is conservative and so few women follow it. Although women's reports are critical to understanding diet and physical activity behavior in pregnancy, self-report alone is not sufficient. Future research would benefit from including providers' perspectives and contrasting such perspectives with what women report. Gathering information from providers should prove useful in better understanding advice in pregnancy, and ideally provide clues as to when and how to intervene to promote optimal nutrition and physical activity among pregnant women.

\subsection{Practice Implications}

These findings suggest some unmet needs and possible strategies. Unmet needs include the need for reaching providers with clear and timely, evidence-based information to share with patients that would dispel myths and help patients sift through myriad advice, as well as helping providers gain skills and self-efficacy to counsel about weight gain and physical activity; the need for all providers in a practice, including staff who come into contact with a patient, to echo the same message; the need to create educational supports or reinforcements so that women can be clear about the recommendations they receive; and the need for women to perceive recommendations are individualized to each person as appropriate. [44] Strategies for reinforcing information might include using social networking technologies such as Text4baby (http://www.text4baby.org/), and/or offering clear, concise handouts that allow the woman to differentiate herself from others, such as a series of questions they mark if relevant to themselves (for example, 'I sometimes eat fish or I drink more than one glass of juice every day', etc.) that is then coupled with advice based on the woman's responses. Useful web resources could include interactive websites such as the United States Department of Agriculture's (USDA) 'Choose My Plate' (a revised version of My Pyramid) (http://www.choosemyplate.gov/pregnancy-breastfeeding.html), that allow for personalization of nutrition intake and are possible tools for achieving individualization without the time consuming requirements of attempting that during a clinical visit. 


\section{Acknowledgments}

We would like to acknowledge Drs. David Savitz, Nancy Dole, Amy Herring, June Stevens, and John Thorp for their roles in the Pregnancy Infection and Nutrition-Postpartum (PINPost) study design and development, as well as Amy Neevel and Shelley Harris for their work on coding the focus group data. We would also like to recognize Gratia Wright of First Research, Inc. and her staff for moderating the focus groups. This research was supported by a grant from The National Institutes of Diabetes and Digestive and Kidney Diseases (Grant No.DK 061981-02).

\section{REFERENCES}

1. World Health Organization. WHO/FAO Expert Consultation, WHO Technical Report. Geneva: WHO; 2002. Diet, Nutrition and the Prevention of Chronic Diseases. Series. Number 916.

2. Prevalence of regular physical activity among adults - United States, 2001 and 2005. MMWR. 2007; 56:1209-1212. [PubMed: 18030281]

3. Blanck HM, Gillespie C, Kimmons JE, Seymour JD, Serdula MK. Trends in fruit and vegetable consumption among US men and women, 1994-2005. Prev Chronic Dis. 2008; 5(2)

4. Brambila-Macias J, Shankar B, Capacci S, Mazzocchi M, Perez-Cueto FJA, Verbeke W, Traill WB. Policy interventions to promote healthy eating: A review of what works, what does not, what is promising. Food Nutr Bull. 2011; 32:365-375. [PubMed: 22590970]

5. Campbell F, Johnson M, Messina J, Guillaume L, Goyder E. Behavioural interventions for weight management in pregnancy: A systematic review of quantitative and qualitative data. BMC Public Health. 2011; 11:491. [PubMed: 21696589]

6. Siega-Riz AM, Bodnar LM, Savitz DA. What are pregnant women eating? Nutrient and food group differences by race. Am J Obstet Gynecol. 2002; 186:480-486. [PubMed: 11904611]

7. Evenson KR, Wen F. National trends in self-reported physical activity and sedentary behaviors among pregnant women: NHANES 1999-2006. Prev Med. 2010; 50:123-128. [PubMed: 20053370]

8. Kaiser L, Allen LH. Position of the American Dietetic Association: nutrition and lifestyle for a healthy pregnancy outcome. J Am Diet Assoc. 2008; 108:553-561. [PubMed: 18401922]

9. Guidelines for Perinatal Care. Elk Grove Village, IL (AAP). 6th ed.. Washington, DC: (ACOG): American Academy of Pediatrics American College of Obstetricians and Gynecologists; 2007.

10. ACOG committee opinion. Exercise during pregnancy and the postpartum period. Number 267 , January 2002. American College of Obstetricians and Gynecologists. Int J Gynaecol Obstet. 2002; 77:79-81. [PubMed: 12053898]

11. Persad VL, Pierotic MA, Guijon FB. Incidence of open neural tube defects in Nova Scotia after folic acid fortification. CMAJ. 2002; 167:241-245. [PubMed: 12186168]

12. Siega-Riz AM, Adair LS, Hobel CJ. Maternal underweight status and inadequate rate of weight gain during the third trimester of pregnancy increases the risk of preterm delivery. J Nutr. 1996; 126:146-153. [PubMed: 8558295]

13. Thompson JM, Wall C, Becroft DM, Robinson E, Wilder CJ, Mitchell EA. Maternal dietary patterns in pregnancy and the association with small-for-gestational-age infants. Br J Nutr. 2010; 103:1665-1673. [PubMed: 20211035]

14. Siega-Riz AM, Herring AH, Carrier K, Evenson KR, Dole N, Deierlein A. Sociodemographic, perinatal, behavioral, and psychosocial predictors of weight retention at 3 and 12 months postpartum. Obesity. 2010; 18:1996-2003. [PubMed: 20035283]

15. Abrams B, Altman SL, Pickett KE. Pregnancy weight gain: Still controversial. Am J Clin Nutr. 2000; 71:1233S-1241S. [PubMed: 10799396]

16. Exercise during pregnancy and the postpartum period. ACOG Technical Bulletin Number 189-February 1994. Int J Gynaecol Obstet. 1994; 45:65-70. [PubMed: 7913067]

17. Melzer K, Schutz Y, Boulvain M, Kaser B. Physical activity and pregnancy: Cardiovascular adaptations, recommendations and pregnancy outcomes. Sports Med. 2010; 40:493-507. [PubMed: 20524714]

18. Redden SL, Lamonte MJ, Freudenheim JL, Rudra CB. The association between gestational diabetes mellitus and recreational physical activity. Matern Child Health J. 2011; 15:514-519. [PubMed: 20213490] 
19. Bung P, Artal R. Gestational diabetes and exercise: A survey. Semin Perinatol. 1996; 20:328-333. [PubMed: 8888458]

20. Dempse JC, Butler CL, Williams MA. No need for a pregnant pause: Physical activity may reduce the occurrence of gestational diabetes mellitus and preeclampsia. Exercise and Sport Sciences Reviews. 2005; 33:141-149. [PubMed: 16006822]

21. Sorensen TK, Williams MA, Lee IM, Dashow EE, Thompson ML, Luthy DA. Recreational physical activity during pregnancy and risk of preeclampsia. Hypertension. 2003; 41:1273-1280. [PubMed: 12719446]

22. Evenson KR, Siega-Riz AM, Savitz DA, Leiferman JA, Thorp JM Jr. Vigorous leisure activity and pregnancy outcome. Epidemiology. 2002; 13:653-659. [PubMed: 12410006]

23. Clapp JF 3rd. Exercise during pregnancy. A clinical update. Clin Sports Med. 2000; 19:273-286. [PubMed: 10740759]

24. Fowles ER. Comparing pregnant women's nutritional knowledge to their actual dietary intake. MCN Am J Matern Child Nurs. 2002; 27:171-177. [PubMed: 12015445]

25. Watts V, Rockett H, Baer H, Leppert J, Colditz G. Assessing diet quality in a population of lowincome pregnant women: A comparison between Native Americans and whites. Matern Child Health J. 2007; 11:127-136. [PubMed: 17191147]

26. Petersen AM, Leet TL, Brownson RC. Correlates of physical activity among pregnant women in the United States. Med Sci Sports Exerc. 2005; 37:1748-1753. [PubMed: 16260976]

27. Evenson KR, Savitz DA, Huston SL. Leisure-time physical activity among pregnant women in the US. Paediatr Perinat Epidemiol. 2004; 18:400-407. [PubMed: 15535815]

28. Chasan-Taber L, Schmidt MD, Pekow P, Sternfield B, Manson J, Markison G. Correlates of physical activity in pregnancy among Latina women. Matern Child Health J. 2007; 11:353-363. [PubMed: 17345155]

29. Jackson RA, Stotland NE, Caughey AB, Gerbert B. Improving diet and exercise in pregnancy with Video Doctor counseling: A randomized trial. Patient Educ Couns. 2011; 83:203-209. [PubMed: 21459255]

30. Patton, MQ. Qualitative Evaluation and Research Methods. 2nd Edition.. Thousand Oaks, CA: Sage Publications; 1990.

31. Madriz, E. Focus groups in feminist research. In: Denzin, N.; Lincoln, Y., editors. Handbook of qualitative research. Vol. 2000. Thousand Oaks, CA: Sage Publications; p. 835-850.

32. Marin G, Sabogal F, Vanoss Marin B, Otero-Sabogal R, Perez-Stable EJ. Development of a short acculturation scale for Hispanics. Hispanic Journal of Behavioral Sciences. 1987; 9:183-205.

33. Miles, M.; Huberman, A. Qualitative data analysis: An expanded sourcebook. 2nd ed.. Thousand Oaks, CA: Sage Publications; 1994.

34. Mirowsky J, Ross CE. Mexican culture and its emotional contradictions. J Health Soc Behav. 1984; 25:2-13. [PubMed: 6725922]

35. Brodie, M.; Steffenson, A.; Valdez, J.; Levin, R.; Suro, R. 2002 National Survey of Latinos: Summary of findings. Washington, DC: Pew Hispanic Center, Kaiser Family Foundation; 2002. (report \#3300).

36. Clarke PE, Gross H. Women's behaviour, beliefs and information sources about physical exercise in pregnancy. Midwifery. 2004; 20:133-141. [PubMed: 15177856]

37. Krans EE, Gearhart JG, Dubbert PM, Klar PM, Miller AL, Replogle WH. Pregnant women's beliefs and influences regarding exercise during pregnancy. J Miss State Med Assoc. 2005; 46:6773. [PubMed: 15822648]

38. Bauer PW, Broman CL, Pivarnik JM. Exercise and pregnancy knowledge among healthcare providers. J Womens Health (Larchmt). 2010; 19:335-341. [PubMed: 20113144]

39. Stotland NE, Gilbert P, Bogetz A, Harper CC, Abrams B, Gerbert B. Preventing excessive weight gain in pregnancy: How do prenatal care providers approach counseling? J Womens Health. 2010; 19:807-814.

40. Entin P, Munhall K. Recommendations regarding exercise during pregnancy made by private/small group practice obstetricians in the United States. J Sports Sci \& Med. 2006; 5:449-458. 
41. Williford HN, Barfield BR, Lazenby RB, Olson MS. A survey of physicians' attitudes and practices related to exercise promotion. Prev Med. 1992; 21:630-636. [PubMed: 1438111]

42. Lyerly AD, Mitchell LM, Armstrong EM, Harris LH, Kukla R, Kupperman M, Little MO. Risks, values, and decision making surrounding pregancy. Obstet Gynecol. 2007; 109:979-984. [PubMed: 17400862]

43. Jasper JD, Goel R, Einarson A, Gallo M, Koren G. Effects of framing on teratogenic risk perception in pregnant women. Lancet. 2001; 358:1237-1238. [PubMed: 11675064]

44. Campbell MK, DeVellis BM, Strecher VJ, Ammerman AS, DeVellis RF, Sandler RS. Improving dietary behavior: The effectiveness of tailored messages in primary care settings. Am J Public Health. 1994; 84:783-787. [PubMed: 8179049] 


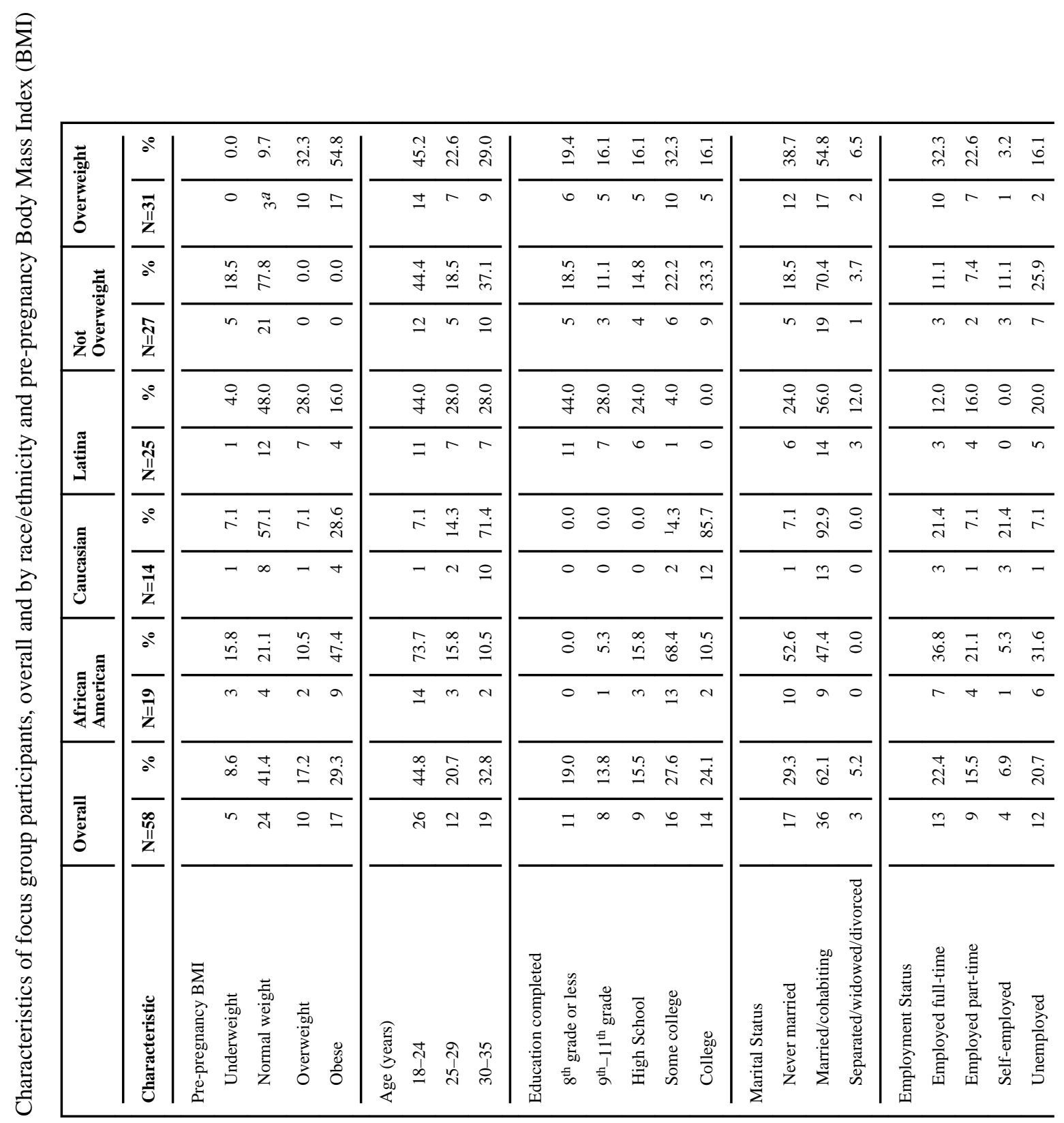




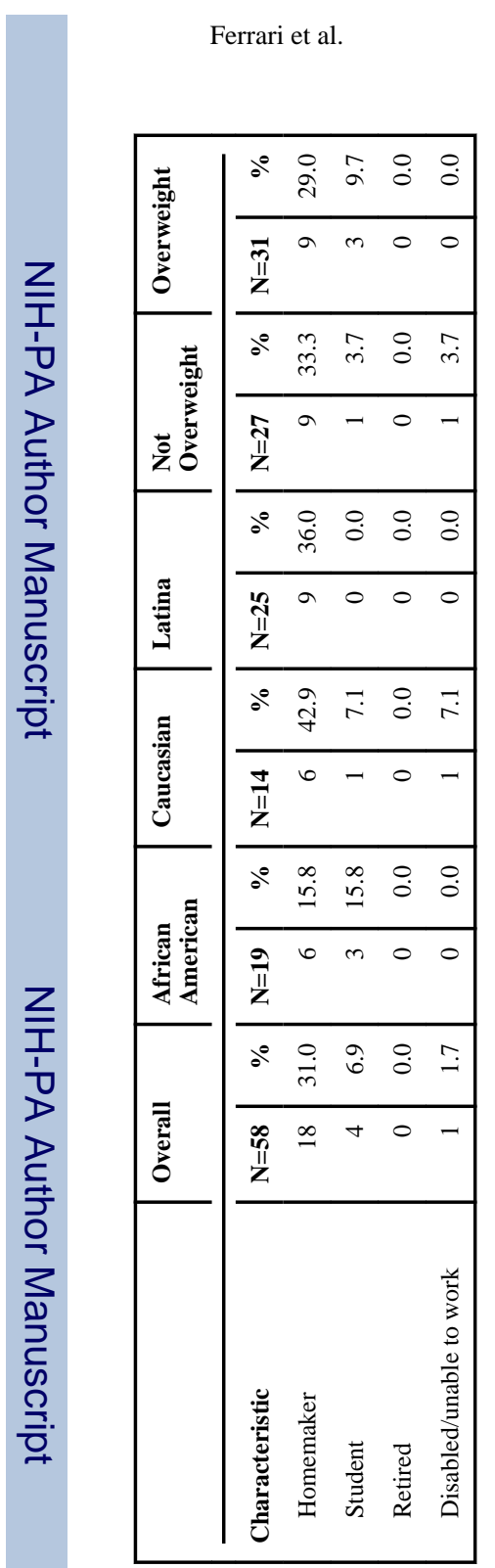

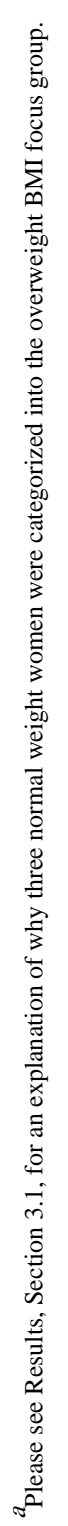

Page 14

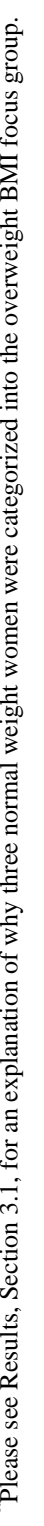

